\title{
Molecularly Distinct NLRP3 Inducers Mediate Diverse Ratios of Interleukin-1 $\beta$ and Interleukin-18 from Human Monocytes
}

\author{
Kristine Midtbö $\mathbb{D}^{1,2}$ Daniel Eklund $\mathbb{D}^{1,2}$ Eva Särndahl $\mathbb{D}^{1,2}$ and Alexander Persson $\mathbb{D}^{1,2}$ \\ ${ }^{1}$ School of Medical Sciences, Faculty of Medicine and Health, Örebro University, SE-701 82 Örebro, Sweden \\ ${ }^{2}$ Inflammatory Response and Infection Susceptibility Centre (iRiSC), Faculty of Medicine and Health, Örebro University, \\ SE-701 82 Örebro, Sweden
}

Correspondence should be addressed to Kristine Midtbö; kristine.midtbo@oru.se

Received 8 July 2020; Revised 10 September 2020; Accepted 29 September 2020; Published 22 October 2020

Academic Editor: Young-Su Yi

Copyright $\odot 2020$ Kristine Midtbö et al. This is an open access article distributed under the Creative Commons Attribution License, which permits unrestricted use, distribution, and reproduction in any medium, provided the original work is properly cited.

Inflammasomes cleave and activate interleukin- (IL-) $1 \beta$ and IL-18 which have both shared and unique biological functions. IL- $1 \beta$ is an important mediator of the acute phase response to infections and tissue damage, whereas IL-18 takes part in activation and tailoring of the adaptive immune response. While IL- $1 \beta$ has served as the prototypic indicator of inflammasome activation, few studies have compared the potential differences in IL-1 $\beta$ and IL-18 production during inflammasome activation. Since these cytokines partake in different immune pathways, the involvement of inflammasome activity in different conditions needs to be described beyond IL- $1 \beta$ production alone. To address a potential heterogeneity in inflammasome functionality, ATP, chitosan, or silica oxide $\left(\mathrm{SiO}_{2}\right)$ were used to induce NLRP3 inflammasome activation in THP-1 cells and the subsequent outcomes were quantified. Despite using doses of the inflammasome inducers yielding similar release of $\mathrm{IL}-1 \beta, \mathrm{SiO}_{2}$-stimulated cells showed a lower concentration of released IL-18 compared to ATP and chitosan. Hence, the cells stimulated with $\mathrm{SiO}_{2}$ responded with a distinctly different IL-18 : IL- $1 \beta$ ratio. The difference in the IL-18 : IL- $1 \beta$ ratio for $\mathrm{SiO}_{2}$ was constant over different doses. While all downstream responses were strictly dependent on a functional NLRP3 inflammasome, the differences did not depend on the level of gene expression, caspase-1 activity, or pyroptosis. We suggest that the NLRP3 inflammasome response should be considered a dynamic process, which can be described by taking the ratio between IL- $1 \beta$ and IL-18 into account and moving away from an on/off perspective of inflammasome activation.

\section{Introduction}

IL-1 $\beta$ and IL-18 are crucial for mounting a potent proinflammatory response and directing subsequent immune responses. Activation of interleukin- (IL-) $1 \beta$ and IL-18 into their biologically active forms require the formation of a multiprotein complex called inflammasome [1]. Today, several inflammasomes have been described, with the nomenclature depending on the main Nod-like receptor (NLR) involved. Upon activation, the NLRs oligomerize to form the core of the inflammasome complex by recruiting the adaptor protein apoptosis-associated speck-like protein containing a CARD (ASC). ASC in turn recruits and activates the serine protease caspase- 1 that is the effector caspase responsible for the bioactivation of IL-1 $\beta$, IL-18, and gasdermin D (GSDMD) $[2,3]$.
Due to the inflammatory potency in the cytokines regulated by inflammasomes, activation of the inflammasome is under strict regulation and requires two distinct signals. Signal one upregulates inflammasome components and the proform of IL-1 $\beta$ [3], while the second signal can then activate a specific NLR, initiating the formation of the inflammasome complex. Whereas many NLRs such as AIM2 and NLRC4 initiate inflammasome formation as a response to specific agonists, the NLRP3 inflammasome can be activated by a broad range of stimuli related to both pathogens, cellular stress and tissue damage. The NLRP3 inflammasome is by far the most studied inflammasome, and the vast array of potential inducers renders it unlikely that the NLRP3 receptor directly interacts with the agonists $[4,5]$. Among the characterized inflammasome inducers are adenosine tri- 
phosphate (ATP), a nucleotide and well-known danger signal which activates NLRP3 through $\mathrm{K}^{+}$efflux after binding to the ATP receptor P2X7 [6-9]. Chitosan, a family of linear polysaccharides found in fungal cell walls, is believed to activate the NLRP3 by lysosomal disruption [10], $\mathrm{K}^{+}$efflux, and/or through induction of reactive oxygen species $[11,12]$. Inorganic silica oxide crystals $\left(\mathrm{SiO}_{2}\right)$ have been reported to activate the NLRP3 inflammasome through lysosome disruption, induction of mitochondrial damage [13, 14], or alternatively through the scavenger receptor SR-B1 [15].

Production of IL- $1 \beta$ is often used as the proxy for describing inflammasome activation. However, using this benchmark indicates that inflammasome activation is a static mechanism, and the possibility of different signaling pathways inducing different effects on the functional aspects following inflammasome activation are generally not discussed. Active IL-1 $\beta$ and IL-18 share several functions as proinflammatory cytokines, but they also have unique properties resulting in distinct immunological profiles. IL- $1 \beta$ is a pyrogen that induces fever, which is a property that IL-18 lack $[16,17]$. Other features of IL- $1 \beta$ include neutrophil mobilization, acute phase protein production, Th17 differentiation, and enhanced antigen presentation and glycolytic rate in Th17 cells and macrophages [16-20]. On the other hand, IL-18 promotes Th1 or Th2 differentiation, depending on the cytokine environment [21], and induces IFN- $\gamma$ production from Th1 cells and NK-cells as well as IL-13 and IL-4 from basophils and mast cells [22].

IL- $1 \beta$ and IL-18 also contribute to the pathogenesis of many diseases, but they play different roles. Since IL- $1 \beta$ promotes Th17 differentiation, IL- $1 \beta$ can be related to many Th17-driven diseases, including rheumatoid arthritis, multiple sclerosis, and psoriasis [23-25]. IL-1 $\beta$ also take part in the pathogenesis of gout, osteoarthritis, inflammatory bowel disease, pericarditis, macrophage activating syndrome, chronic obstructive pulmonary disease (COPD) [26-31], and chronic systemic inflammatory conditions, like cryopyrin-associated periodic syndrome (CAPS), adult-onset Still's disease, and familial Mediterranean fever (FMF) [26, 32, 33]. On the other hand, IL-18 is strongly related to IFN- $\gamma$ and Th1/Th2 diseases, like systemic lupus erythematosus, Crohn's disease, and graft versus host rejection $[16,31,34,35]$. IL-18 is also a key player in allergy, atherosclerosis, dermatitis, acute renal ischemia, hepatitis, and heart failure [31, 36-41]. However, even if IL- $1 \beta$ or IL-18 may play a dominant role, both often contribute to the disease but play separate roles at different stages of diseases. Knockout studies in a mouse model of CAPS showed that loss of the IL- $1 \beta$ or IL- 18 receptor was differently beneficial in young and old mice [42]. Additionally, FMF have shown to be correlated to elevated levels of IL-18, while IL- $1 \beta$ play a dominant role during inflammatory flare ups that are treated with IL- $1 \beta$ inhibition $[43,44]$. Several approved therapies target the IL-1 receptor (IL-1R) or free circulating IL- $1 \beta$, including the IL-1R antagonist anakinra (Kineret ${ }^{\circledR}$ ). This have been proven to be successful in many cases but since targeting IL- $1 \beta$ does not inhibit the effect of IL-18, it is not surprising that certain conditions are unaffected by the treatment [45], and recently, efforts have been made to investigate the effectiveness of instead blocking IL-18 in certain inflammatory diseases [41, 46, 47]. Since IL- $1 \beta$ and IL- 18 contribute diversely to different diseases, it is unlikely that inflammasome activation is a static on/off process. Consequently, when studying inflammasome functionality, the activity may need to be described beyond IL- $1 \beta$ production. This study was therefore designed to describe the outcome of NLRP3 inflammasome activation and the functional effects of diverse inflammasome inducers.

\section{Materials and Methods}

2.1. Cell Culture. THP-1 cells were cultured in RPMI medium 1640 (Gibco, Thermo Fisher Scientific, Waltham, MA cat. nr:31870-025) supplemented with $10 \%$ fetal bovine serum (FBS), Hepes $(10 \mathrm{mM})$, sodium pyruvate $(1 \mathrm{mM})$, GlutaMax $(2 \mathrm{mM})$, glucose $(2.5 \mathrm{~g} / \mathrm{L})$, and penicillin-streptomycin (PEST, $100 \mathrm{U} / \mathrm{mL}$ ) all from Gibco (Thermo Fisher Scientific). The THP-1 cell lines used are THP-1 null cells (thp-null), THP-1 defNLRP3 cells (rhp-dnlp), THP-1 defASC cells (thp-dasc), THP-1 defCASP1 (thp-dcasp1), and THP-1 Xblue ${ }^{\mathrm{TM}}-\mathrm{MD} 2-$ CD14 cells (thp-mdcdsp) which were all acquired from InvivoGen (San Diego, CA). The THP-1 cells were kept undifferentiated as a model of human monocytes.

\subsection{Isolation of Primary Human Monocytes. Human CD14 $4^{+}$} cells were isolated from healthy volunteers. PBMCs were separated by density gradient centrifugation on Lymphoprep (Axis-Shield, Oslo, Norway), and the CD14 ${ }^{+}$cells were isolated by magnetic sorting using CD14 MACS microbeads (Miltenyi Biotech, Bergisch Gladbach, Germany) according to the manufacturer's protocol. The cells were cultured in DMEM medium (Lonza BioWhittaker, Thermo Fisher Scientific cat. nr: 11635220) supplemented with $10 \%$ human $\mathrm{AB}$ serum (pooled from five healthy volunteers), L-glutamine $(5 \mathrm{mM})$, sodium pyruvate $(5 \mathrm{mM})$, and glucose $(2.5 \mathrm{~g} / \mathrm{L})$ (Gibco, Thermo Fisher Scientific).

2.3. Experimental Setup. THP-1 cells were seeded into 96- or 24-well plates and primed with lipopolysaccharide- (LPS-) B5 (E. coli serotype 055:K59(B5)H-, $100 \mathrm{ng} / \mathrm{mL}$, or concentrations indicated in Figure 1(d)) for 10 minutes to induce instant priming, followed by additional stimulation with ATP $(5 \mathrm{mM})$, chitosan $(30 \mu \mathrm{g} / \mathrm{mL})$, or $\mathrm{SiO}_{2}(30 \mu \mathrm{g} / \mathrm{mL})$ all from InvivoGen, for $24 \mathrm{~h}$.

Isolated primary human monocytes were primed with $10 \mathrm{ng} / \mathrm{mL}$ LPS prior to stimulation with $10 \mathrm{nM}$ ATP, $10 \mathrm{ng} / \mathrm{mL}$ chitosan, or $10 \mathrm{ng} / \mathrm{mL} \mathrm{SiO}_{2}$.

2.4. Cytokine Measurement. The concentrations of cytokines were measured with enzyme-linked immunosorbent assay (ELISA) for IL-1 $\beta$ (BioLegend, San Diego, CA) and IL-18 (R\&D Systems, Minneapolis, MN) according to the manufacturer's instruction.

2.5. SEAP Reporter Assay. NF- $\kappa \mathrm{B}$ activity was measured indirectly by the SEAP activity from a NF- $\kappa \mathrm{B}$ responsive SEAP reporter gene in the reporter THP-1 xBlue ${ }^{\mathrm{TM}}$ cells. The accumulation of SEAP in the culture medium $24 \mathrm{~h}$ after stimulation was detected with the QUANTI-Blue ${ }^{\mathrm{TM}}$ (InvivoGen) according to the manufacturer's instructions. The OD was 


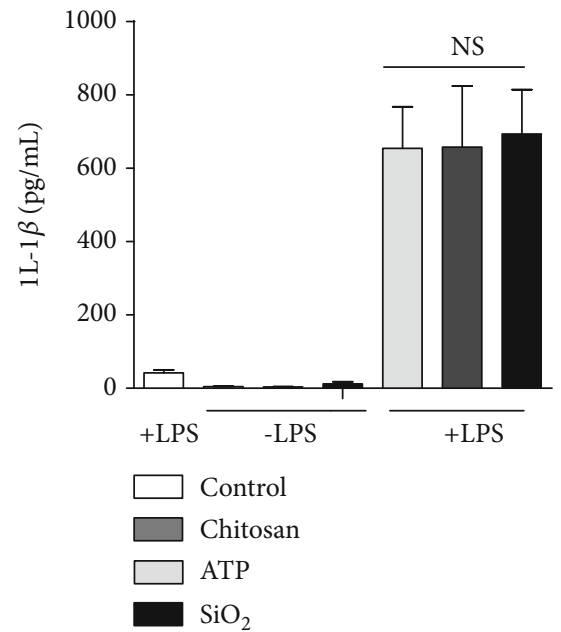

(a)

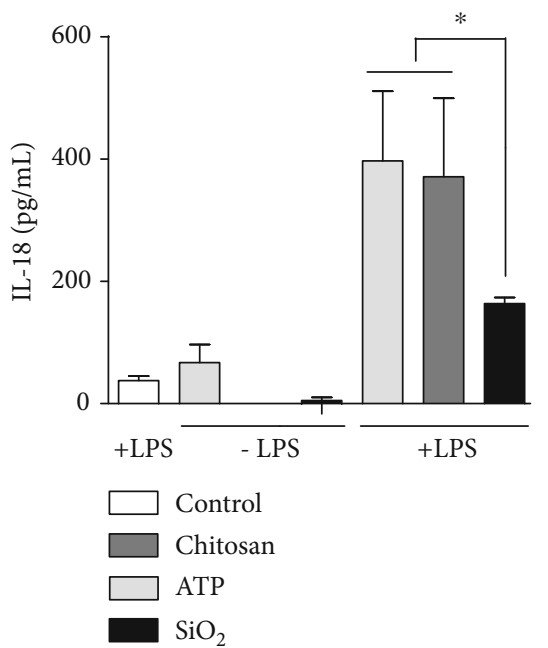

(b)

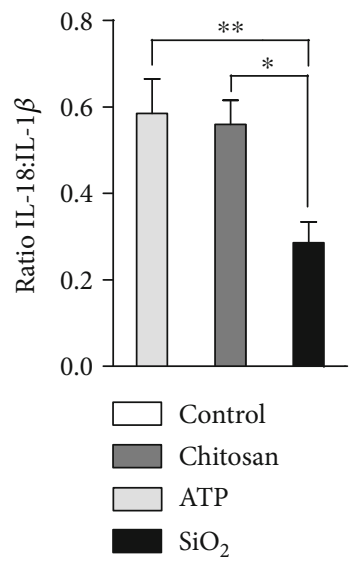

(c)

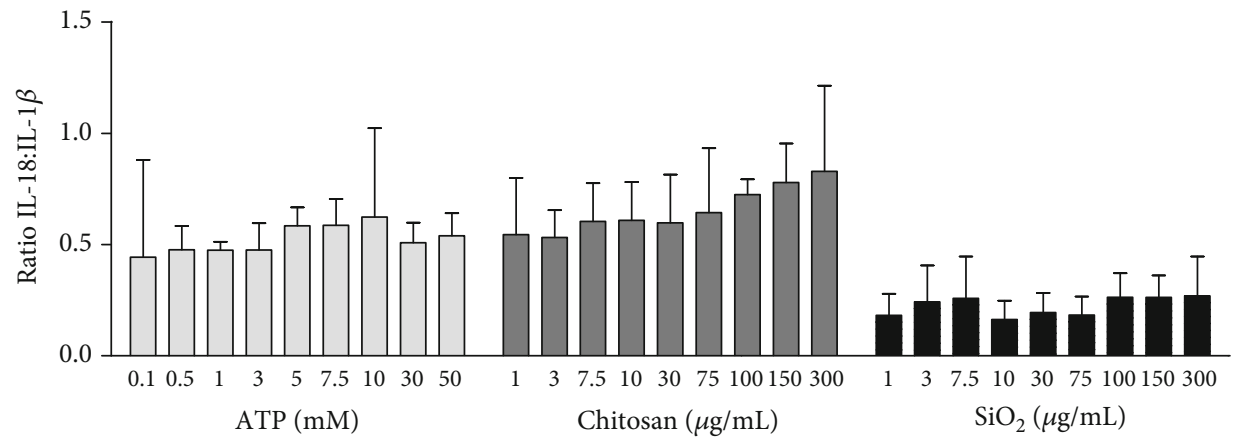

(d)

FIgURE 1: ATP, chitosan, and $\mathrm{SiO}_{2}$ induce diverse IL-18: IL- $1 \beta$ ratio in THP-1 cells. The concentration of released IL- $1 \beta$ (a) and IL-18 (b) in nonprimed (-LPS) and LPS-primed (+LPS, $100 \mathrm{ng} / \mathrm{mL}$ ) cells, $24 \mathrm{~h}$ post stimulation were detected after stimulation with $5 \mathrm{mM} \mathrm{ATP,} 30 \mu \mathrm{g} / \mathrm{mL}$ chitosan, or $30 \mu \mathrm{g} / \mathrm{mL} \mathrm{SiO} 2$. The ratio between IL-1 $\beta$ and IL-18 was calculated for each experiment (c) and after stimulation with increasing doses of ATP $(\mathrm{mM})$, chitosan $(\mu \mathrm{g} / \mathrm{mL})$, or $\mathrm{SiO}_{2}(\mu \mathrm{g} / \mathrm{mL})(\mathrm{d})$. Controls were left untreated (-LPS) or treated with LPS alone (+LPS). Data are shown as mean \pm SD from three-six individual experiments. NS: non significant, ${ }^{*} p<0.05 ;{ }^{* *} p<0.01$.

measured at $635 \mathrm{~nm}$ with Cytation 3 imaging plate reader (BioTek, Winooski, VT).

2.6. FLICA Assay. Activation of caspase-1 was detected with the fluorescent probe FAM-YVAD-FMK (FLICA) from Immunochemistry Technologies (Bloomington, MN cat.nr: OKSA11275), that binds irreversibly to active caspase-1. The cells were stained for $1 \mathrm{~h}$ and washed two times in PBS prior to analysis. The percentage of positive cells was measured by flow cytometry, (Accuri ${ }^{\mathrm{TM}}$ C6, BD, Franklin Lakes, NJ).

2.7. Lactate Dehydrogenase Assay. Cellular rupture was detected using the Pierce ${ }^{\circledR}$ lactate dehydrogenase (LDH) cytotoxicity assay kit (Thermo Scientific) according to the manufacturer's instructions. Data are presented as percentage $\mathrm{LDH}$ calculated from a positive control (100\% lysed cells).
2.8. Western Blotting. Cells used for Western blotting were stimulated in FBS-free media. Cells were lysed in RIPA lysis buffer (Merck Millipore, Burlington, MA), and the protein concentrations were measured using the Micro BCA ${ }^{\mathrm{TM}}$ Protein Assay (Thermo Scientific). The proteins were separated in $8-16 \%$ stain-free TGX gels (Bio-Rad, Hercules, CA), transferred to PVDF membranes (iBlot ${ }^{\circledR} 2$ PVDF regular stacks, Invitrogen, Carlsbad, CA), and analysed by immunoblotting. The primary antibodies used were cleaved IL-1 $\beta$ (1:1000 Cell Signaling Technologies, Danvers, MA. cat.nr:12242), IL-18 (1:1000 Abcam, Cambridge, UK. cat.nr: EPR19954188), and caspase-1 p20 (1:750 Adipogen Life Sciences, cat.nr:AG-20B-0048-C100). Secondary antibodies used were goat anti mouse (1:5000 Abcam cat.nr: Ab6789), rabbit anti goat (1:2000 Dako, Agilent. Santa Clara, CA. cat.nr: P0160), and goat anti-rabbit (1:3000 Invitrogen cat.nr: A11034). Membranes were washed in TBST buffer and analysed with ChemiDoc $^{\mathrm{TM}}$ MP Imaging System (Bio-Rad). 
2.9. Extraction of $m R N A$, Reverse Transcription, and $q P C R$. Cells were lysed in RLT lysis buffer (Qiagen, Hilden, Germany) and drawn through a $22 \mathrm{G}$ needle to homogenize the sample. Total RNA was extracted and purified with the QIAmp RNeasy Mini kit (Qiagen). RNA was quantified using NanoDrop 2000 (Thermo Fisher Scientific), and a High-Capacity cDNA Transcription kit (Thermo Fisher Scientific) was used for the reverse transcription reactions (900 ng of total RNA per $60 \mu \mathrm{L}$ reaction) on a LifePro Thermal Cycler (Bioer, Hangzhou, China). Quantitative realtime PCR was performed using TaqMan assays in QuantStudio 7 Flex PCR (Applied Biosystems, Thermo Fisher Scientific). The TaqMan assays used were IL1B (Hs01555410), IL18 (Hs01038788_m1), NLRP3 (Hs00918082_m1), ASC/PYCARD (Hs01547324_gH), CASP1 (Hs00354836_m1), CASP8 (Hs01018151_m1), HPRT1 (Hs02800695_m1), and TBP (Hs00427620_m1), (all from Thermo Fisher Scientific). A comparative quantification was used, where the quantity of each experimental sample was determined using a standard curve as calibrator samples. Calibrator was prepared from human peripheral blood mononuclear cell (PBMC) cultures stimulated for $48 \mathrm{~h}$ with $1 \mu \mathrm{g} / \mathrm{mL}$ LPS known to express the genes of interest in high abundance. A six-point serially fourfold diluted standard curve was developed from the calibrator by plotting the threshold cycles versus the dilution factor and the data fitted to a straight line, while confirming that the correlation coefficient (R2) for the line was 0.99 or greater. This plot was then used for extrapolating relative expression level information for the same gene of interest in unknown experimental samples. The relative quantity for the gene of interest was normalized to that of a reference gene in the same sample, and then, the normalized numbers was compared between samples. The reference genes used for normalization, HPRT1 and TBP, were selected from four candidate genes using NormFinder R package (MOMA, Aarhus University Hospital, Denmark), where the geometric mean of said genes were used. RNA from stimulated PBMCs was extracted using QIAamp RNA Blood Mini Kit (Qiagen, Hilden, Germany) and converted into cDNA using the above protocols. 384-well plates were prepared using a PIRO Pipetting Robot (Dornier, Lindau, Germany). Cycle threshold (CT) cut-off value was set to 35 cycles. An acceptable coefficient of variation (CV) between duplicates was set to $<15 \%$. Water was used as the negative control.

2.10. Statistics. Statistical analyses were performed using GraphPad Prism 5. $p$ values were assessed using two-tailed Student's $t$-tests and two-way analysis of variance (ANOVA) followed by Bonferroni's posttest. In the figures, ${ }^{*} p<0.05$, ${ }^{* *} p<0.01$, and ${ }^{* * *} p<0.001$. All data shown are mean \pm SD for a minimum of three independent experiments.

\section{Results}

3.1. IL-1 $\beta$ and IL-18 Release Is Mediated by Inflammasome Inducers ATP, Chitosan, or $\mathrm{SiO}_{2}$. To confirm NLRP3 inflammasome activation by ATP, chitosan, and $\mathrm{SiO}_{2}$, respectively, and their dependency on a priming signal, cells were primed with two doses of LPS. Cells stimulated with $1 \mu \mathrm{g} / \mathrm{mL}$ LPS alone showed elevated levels of IL- $1 \beta$, uncharacteristic of a true priming signal and only minor changes in IL- $1 \beta$ and IL-18 production upon stimulation with ATP, chitosan, or $\mathrm{SiO}_{2}$. However, $100 \mathrm{ng} / \mathrm{mL}$ LPS induced only low concentrations of IL- $1 \beta$ and led to significant synergistic effect upon addition of inflammasome inducers (supplementary figure 1). Henceforth, all experiments performed on THP-1 cells were primed with $100 \mathrm{ng} / \mathrm{mL}$ LPS. To investigate potential differences in the response following inflammasome activation, concentrations of the inducers were titrated to doses resulting in similar IL-1 $\beta$ levels released $24 \mathrm{~h}$ after stimulation (supplementary Figure 2. Stimulation of primed cells with $5 \mathrm{mM}$ ATP, $30 \mu \mathrm{g} / \mathrm{mL}$ chitosan, or $30 \mu \mathrm{g} / \mathrm{mL} \mathrm{SiO}{ }_{2}$ induced a significantly increased amount of IL- $1 \beta$ compared to primed controls but showed no significant difference in the concentration of released IL- $1 \beta$ when compared to each other (Figure 1(a)). However, stimulation with $\mathrm{SiO}_{2}$ generated significantly lower levels of IL-18 compared to ATP and chitosan (Figure 1(b)). In terms of ratios, the IL-18 : IL-1 $\beta$ ratio for ATP and chitosan was 1:0.6 in contrast to $1: 0.2$ for $\mathrm{SiO}_{2}$ (Figure 1(c)). The divergent ratio of $\mathrm{SiO}_{2}$ remained constant over a broad range of doses, indicating that the ratio is not sensitive to the amount of inducer (Figure 1(d)).

3.2. Gene Expression of Inflammasome Components in ATP-, Chitosan-, or $\mathrm{SiO}_{2}$-Stimulated Cells. To elucidate whether the differences in IL-18 : IL- $1 \beta$ ratio could be explained by changes in expression levels of inflammasome components and cytokines, gene expression levels of IL1B, IL18, NLRP3, $P Y C A R D$ and CASP1 were analysed. None of the inducers showed any effect on IL1B and IL18 mRNA expression in LPS-primed cells (Figures 2(a) and 2(b)). Chitosan alone increased the CASP1 expression (Figure 2(c)). No significant change was seen for NLRP3 or the potential noncanonical route of activation through CASP8. Furthermore, the inducers showed no own effect on NF- $\kappa$ B activation in unprimed cells. However, chitosan showed a synergistic increase in LPSprimed cells, as measured by utilizing the reporter gene SEAP in a modified THP-1 cell line (Figure 2(d)).

3.3. Activation of Caspase-1 and Induction of Cell Lysis by Diverse Inducers. To further investigate if observed differences in the IL-18 : IL-1 $\beta$ ratio could be attributed to differences in caspase-1 activation, FLICA staining was used to quantify caspase- 1 activity, and the presence of pro- and cleaved caspase-1 was detected by Western blot. Chitosan and $\mathrm{SiO}_{2}$ but not ATP resulted in an increased percentage of FLICA-positive cells compared to primed controls, $24 \mathrm{~h}$ after stimulation (Figure 3(a)). No difference in the protein expression of pro-caspase- 1 could be seen compared to the control for any of the inducers (data not shown), and any tendency of differentiating the expression of pro-IL- $1 \beta$ and cleaved caspase-1 p20 fragment was statistically nonsignificant (Figures 3(c) and 3(d)). Furthermore, an LDH assay was used to examine the percentage of cell lysis $24 \mathrm{~h}$ after stimulation with the inducers. Chitosan and $\mathrm{SiO}_{2}$ stimulation resulted in increased LDH release compared to the primed control (Figure 3(b)). The only significant difference between the inducers with regard to both LDH release and FLICA staining was found between $\mathrm{ATP}$ and $\mathrm{SiO}_{2}$. The $\mathrm{LDH}$ results 


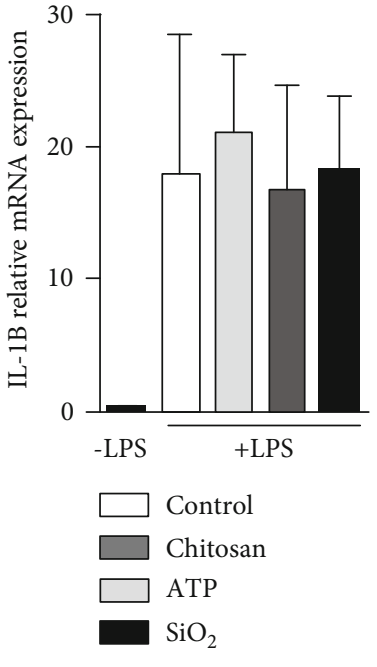

(a)

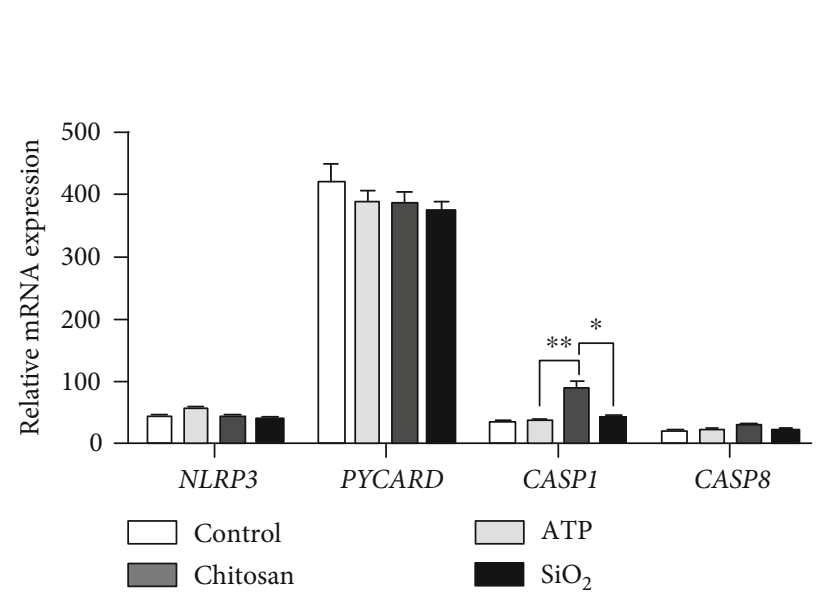

(c)

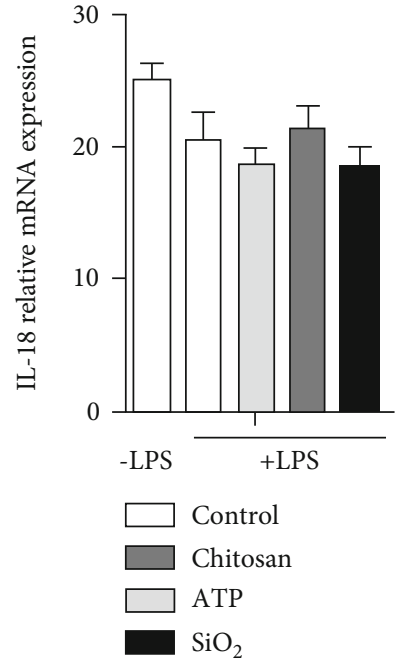

(b)

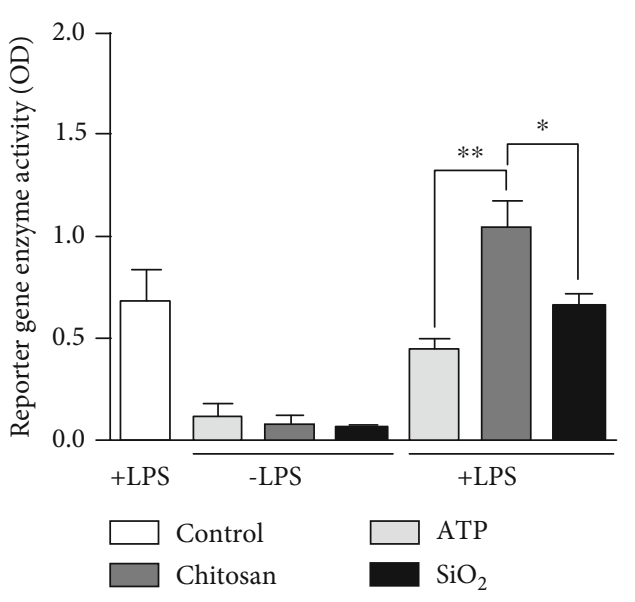

(d)

FIGURE 2: Gene expression of inflammasome components and cytokines do not correlate with the IL-18: IL- $1 \beta$ ratio. The relative mRNA expression of IL1B (a), IL18 (b), and the NLRP3 inflammasome components (c) NLRP3, ASC, and CASP1 as well as CASP8 were measured $24 \mathrm{~h}$ after cells primed with LPS were stimulated with ATP, chitosan, or $\mathrm{SiO}_{2}$ using qPCR. NF- $\kappa$ B activation was detected indirectly by measuring the activation of the reporter gene SEAP (d). Controls were treated with LPS alone. Data are shown as mean \pm SD from six individual experiments. ${ }^{*} p<0.05 ;{ }^{* *} p<0.01$.

were validated by annexin $\mathrm{V}$ and 7AAD staining detected by flow cytometry (data not shown).

3.4. Release of Cytokines in Inflammasome-Deficient Cells by Diverse Inducers. The relevance of the NLRP3 inflammasome components for the response to the inducers were investigated using THP-1 knockout cells, lacking functional NLRP3, ASC, or caspase-1. Absence of NLRP3, ASC, and caspase-1, respectively, completely attenuated the release of IL- $1 \beta$ and IL-18 (Figures 4(a) and 4(b)). While the inducers stimulated the release of other cytokines, not directly regulated by the inflammasome, (supplementary table I), this release was attenuated in inflammasome-deficient cells for several of the cytokines (supplementary figure 3), showing a primary dependency on inflammasome activation for subsequent responses.
3.5. IL-18: Il-1 $\beta$ Ratios in Primary Monocytes. Lastly, in order to investigate whether the results found in the THP-1 cell line is reflected in primary human cells, monocytes were isolated from healthy volunteers, primed with LPS and stimulated with ATP, chitosan, or $\mathrm{SiO}_{2}$. As with the THP-1 cells, the doses of inducers were titrated to achieve the same concentration of released IL-1 $\beta$ (supplementary Figure 2). However, a lower dose of LPS was required for priming primary human monocytes compared to THP-1 cells. At this dose, a synergistic effect by the inflammasome inducers was observed without risking the activation of the noncanonical pathway of inflammasome activation as previously reported by Gaidt et al. [48]. However, unlike the THP-1 cells, the concentrations of released IL-18 were higher than for IL- $1 \beta$ for all inducers. Although not significant, monocytes responded with a similar pattern, as seen in THP-1 cells, with regard to IL-18 : IL-1 $\beta$ ratios (Figures 5(a) and 5(b)). 


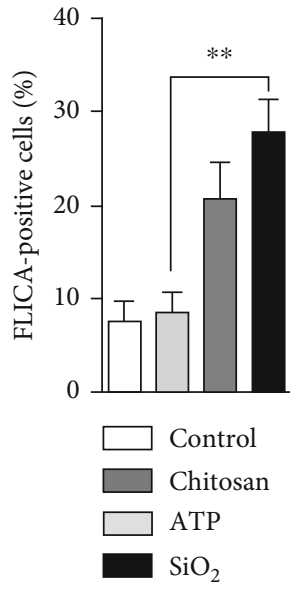

(a)

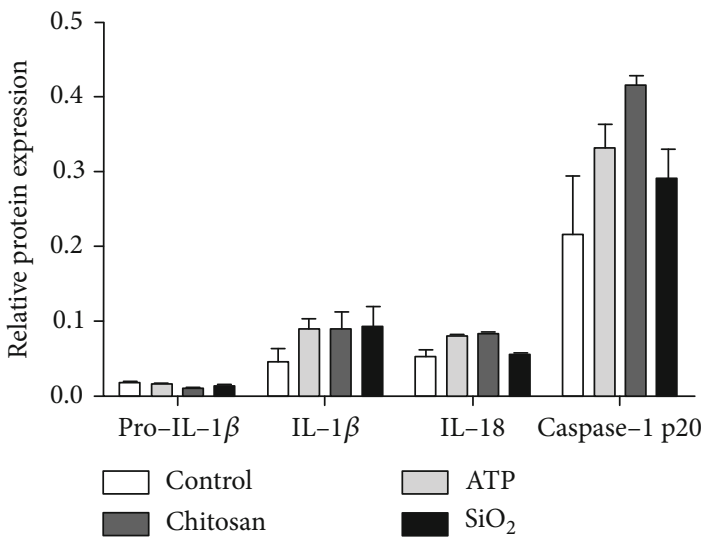

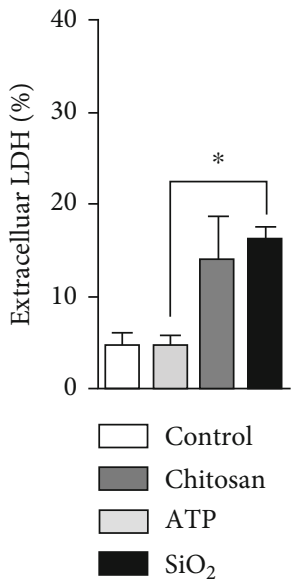

(b)

(c)

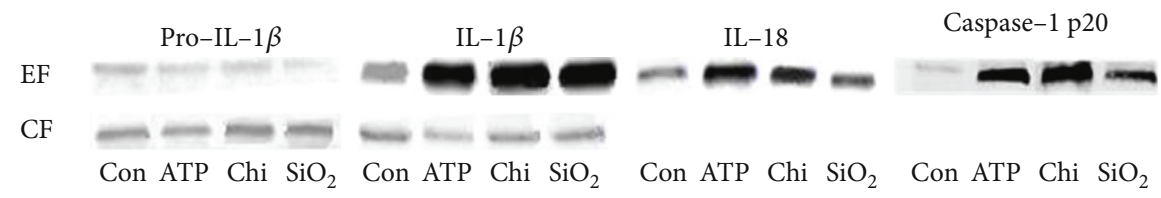

(d)

FIGURE 3: Caspase-1 activity and cell lysis do not correlate with the IL-1 $\beta$ : IL-18 ratio. Caspase-1 activity was measured by flow cytometry using the FLICA probe (a), while LDH assay was used as a measure of cell rupture (b). Pro-IL- $1 \beta$, cleaved IL- $1 \beta$, IL-18, and caspase- 1 p20 were detected in the extracellular fraction (EF) or cellular lysate as the cellular fraction (CF) by Western blot. The blot density was normalized against the total protein load to calculate the fraction (c). Representative blots are shown in (d). Measurement took place $24 \mathrm{~h}$ after stimulation, and controls were treated with LPS alone. Data are shown as mean \pm SD from six individual experiments. ${ }^{*} p<0.05$; ${ }^{* *} p<0.01$.

\section{Discussion}

NLRP3 inflammasome can be activated by numerous inducers and several pathways leading to inflammasome activation have been described. In this study, the differences in functional outcome of three diverse inflammasome inducers, ATP, chitosan, and $\mathrm{SiO}_{2}$, with respect to their ability to activate the inflammasome to produce IL- $1 \beta$ and IL-18 were investigated. Despite inducing the same level of IL- $1 \beta$ release, the studied inducers showed a diverse ability to induce the production of IL-18. This diverse IL-18 : IL- $1 \beta$ ratio remained throughout a broad span of doses. While all three inducers showed a strict dependency on priming with LPS and a functional NLRP3 inflammasome, differences could not be attributed to caspase-1 activity, induction of gene expression, or their ability to induce cell death. Furthermore, these inducers exerted diverse effects on other inflammatory cytokines in an NLRP3-dependent fashion. This strengthens the idea that inflammasome activity is highly contextdependent and flexible process that cannot be regarded as an on/off process.

In this study, $\mathrm{SiO}_{2}$ stimulation resulted in a lower release of IL-18 compared to ATP and chitosan, which also resulted in a different IL-18 : IL- $1 \beta$ ratio. Since IL- $1 \beta$ and IL-18 have both shared and unique biological properties, the ratio between them may impact the overall immunological profile, including Th1 or Th17 differentiation [16]. The inducers used in this study represent the broad range of different 


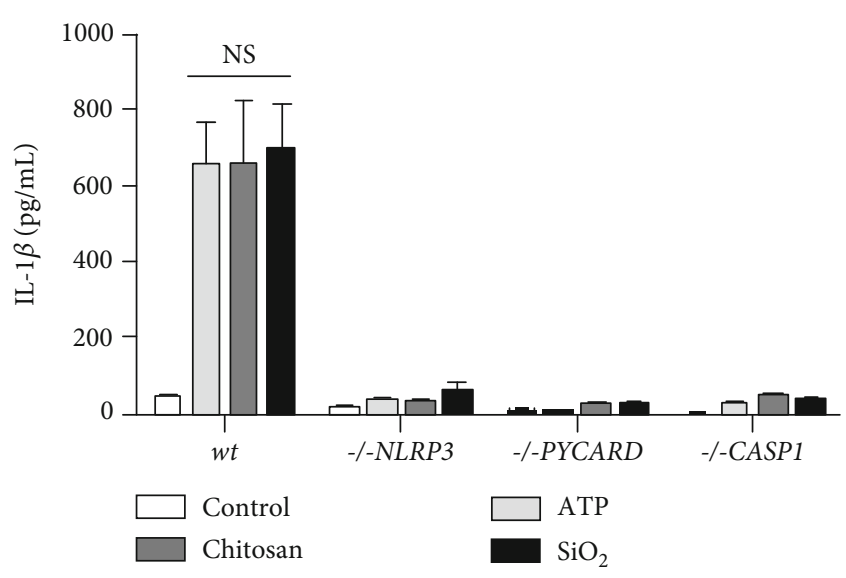

(a)

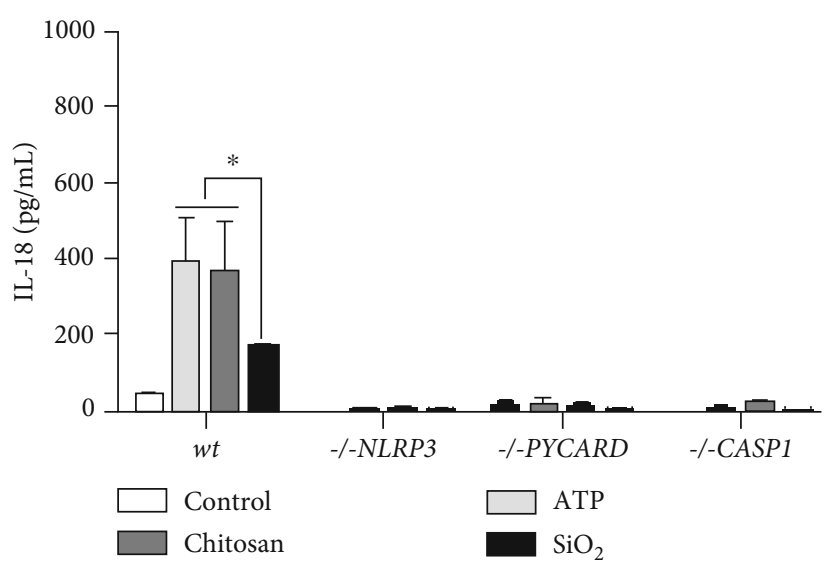

(b)

FIgURE 4: Release of IL- $1 \beta$ and IL-18, induced by ATP, chitosan, or $\mathrm{SiO}_{2}$ is strictly NLRP3 inflammasome dependent. The concentrations of released IL-1 $\beta$ (a) and IL-18 (b) were measured from LPS-primed THP-1 monocytes with functional NLRP3 inflammasome (wt) and inflammasome-deficient THP-1 cells, lacking either functional NLRP3 (-/-NLRP3), ASC (-/-PYCARD), or caspase-1 (-/- CASP1) following stimulation with ATP, chitosan, or $\mathrm{SiO}_{2}$. Controls were treated with LPS alone. Data are shown as mean \pm SD from six individual experiments. NS: nonsignificant. ${ }^{*} p<0.05$.

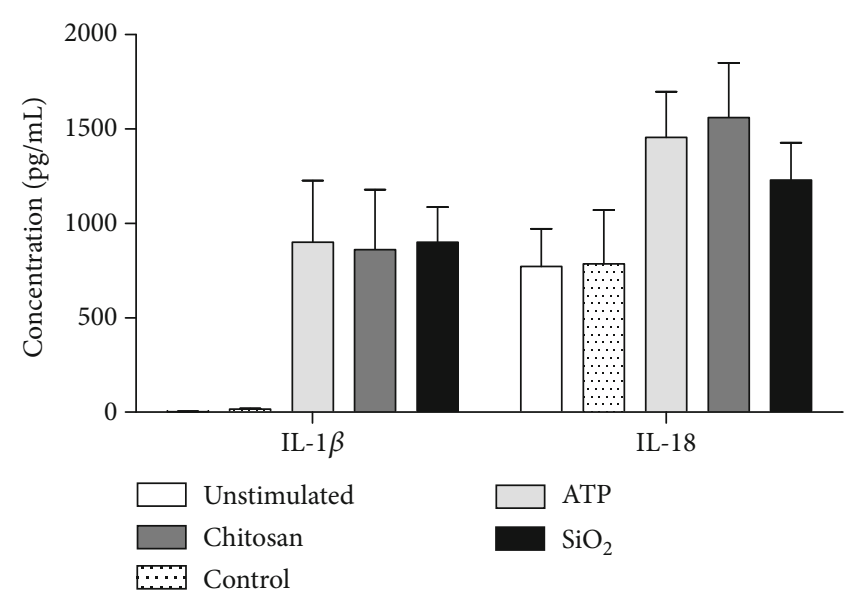

(a)

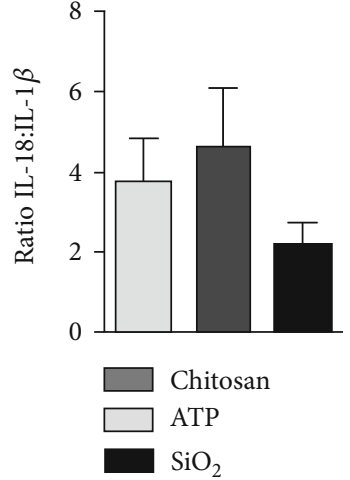

(b)

Figure 5: ATP, chitosan, and $\mathrm{SiO}_{2}$ induce release of IL- $1 \beta$ and IL-18 in primary monocytes. Primary human monocytes were isolated from peripheral blood from healthy volunteers. The release of IL- $1 \beta$ and IL-18 was measured after stimulation with $10 \mathrm{nM}$ ATP, $10 \mathrm{ng} / \mathrm{mL}$ chitosan, or $10 \mathrm{ng} / \mathrm{mL} \mathrm{SiO}$ from LPS-primed cells $(10 \mathrm{ng} / \mathrm{mL})$ (a). The IL-18 : IL- $1 \beta$ ratios were calculated (b). Data are shown as mean \pm SD from six individual experiments.

stimuli that the NLRP3 inflammasome can respond to ATP is a DAMP involved in autoinflammatory diseases that arise during sterile inflammatory conditions, including CAPS and FMF, conditions involving both IL- $1 \beta$ and IL-18 [49, 50]. Meanwhile, PAMP-induced inflammation may result in Th1, Th2, or Th17 differentiation, depending on the type of pathogen. Fungal chitosan is a PAMP, and clearance of fungi requires activation of Th1 and Th17 and therefore relies on both IL-1 $\beta$ and IL-18 for efficient clearance [16, $51,52] . \mathrm{SiO}_{2}$ on the other hand induces inflammation as a result of environmental exposure and its low IL-18 : IL-1 $\beta$ ratio can also be viewed as an IL- $1 \beta$ dominant inflammation. $\mathrm{SiO}_{2}$ and other particle exposure are known to be correlated with an increased risk of systemic autoinflammatory diseases, cardiovascular events, and lung disease, such as COPD, which are also strongly related to IL-1 $\beta$ [53-56], and that can benefit from IL-1 $\beta$ blocking treatment [57].

Furthermore, the importance of ratios between polarizing cytokines in cell differentiation and the immunological response have been suggested by Zielinski et.al [18], but the relevance of the ratio between IL-1 $\beta$ and IL- 18 have not been studied. The diverse ratios also show that the NLRP3 inflammasome activity is not static and can give a dynamic response, depending on the inducers. This flexibility of the NLRP3 inflammasome has previously been implicated by Schmidt and Lenz [58] and Bezbradica et al. [59], who demonstrate that the NLRP3 inflammasome show a greater response when stimulated with exogenous PAMPs than 
endogenous DAMPs. In the current study, several attempts were made to elucidate a mechanism that could explain the difference in IL-18 : IL-1 $\beta$ ratio for $\mathrm{SiO}_{2}$, but no sufficient models were found. Nonetheless, differential regulation of $I L 1 B$ and IL18 mRNA expressions could be excluded as a mechanism, since the inducers did not affect the mRNA expression. Only LPS affected the IL1B expression, with a 100 -fold increase compared to untreated controls, while IL18 was constitutively expressed, as shown previously by Puren et al. [60] and Zhu and Kanneganti [61]. Likewise, mRNA expression of the inflammasome components also failed to explain why $\mathrm{SiO}_{2}$ gives a diverse ratio, as both chitosan and $\mathrm{SiO}_{2}$ show a lower PYCARD mRNA expression and chitosan alone affect the CASP1 expression. Besides NLRP3, PYCARD, and CASP1, the mRNA expression of CASP8 was also examined as caspase- 8 has been previously shown to assist in inflammasome activation $[48,62]$ but its role here cannot be confirmed.

Additionally to IL- $1 \beta$ and IL- 18 , the release of other cytokines, not directly regulated by the inflammasome, were measured and are summarized in supplementary table I. Stimulation with the inducers resulted in an increased release of several cytokines compared to primed control, of which chitosan significantly affected IL-6 and TNF compared to ATP and $\mathrm{SiO}_{2}$ stimulation. The released IL-6 and TNF is likely a secondary response to the inducers, mediated indirectly by IL- $1 \beta$ or IL-18 as the release did not occur in the THP-1 knockout cells deficient of NLRP 3 and caspase-1 (supplementary figure 3 ). However, this secondary response could not clearly be correlated to the IL-18 : IL-1 $\beta$ ratio. Furthermore, the difference in IL-18: IL- $1 \beta$ ratio could not be attributed to the caspase- 1 activity as chitosan and $\mathrm{SiO}_{2}$, but not ATP, showed a higher percentage of cells positive for FLICA $24 \mathrm{~h}$ after stimulation, when compared to LPS-primed controls. A difference in time kinetics between the three inducers may lead to a bias in FLICA-positive cells at $24 \mathrm{~h}$. However, the ratios described in this paper are derived from the accumulated production and release of inflammasome-activated cytokines at $24 \mathrm{~h}$ in order to dilute the effect of inflammasome time kinetics. Next, as induction of cell death and the subsequent unspecific membrane leakage may affect the amounts of cytokines available for extracellular detection, extracellular $\mathrm{LDH}$ was used to measure cell lysis. ATP induced the release of IL- $1 \beta$ without an increase of cell lysis compared to the controls. It is therefore likely that ATP induces GSDMD pores while escaping a pyroptotic fate, which have been demonstrated as a possible alternative to pyroptosis $[48,63]$. Furthermore, the percentage of lysed cells did not reach over $30 \%$ after stimulation with chitosan and $\mathrm{SiO}_{2}$ which means that the majority of the cells are still intact after $24 \mathrm{~h}$. However, since chitosan and $\mathrm{SiO}_{2}$ both increased the percentage of lysed cells, the difference in the IL-18 : IL- $1 \beta$ ratio cannot be explained by pyroptosis.

\section{Conclusions}

Taken together, our data suggest that the different inflammasome inducers lead to a diverse functional outcome that goes beyond the direct production of the hallmark cytokine IL-1 $\beta$, irrespective of gene expression, cell lysis, and caspase- 1 activity. The differential regulation of IL-1 $\beta$ and IL-18 cleavage in human cells need further elucidation, and given the fact that the NLRP3 inflammasome reacts to such a vast array of molecules and have been implicated in numerous, clinically distant diseases, a more holistic approach to studying inflammasome activation under different conditions is warranted. In conclusion, this study shows that the NLRP3 inflammasome is capable of directly tailoring the specific response to a particular stimulus. By taking into account the ratio between IL- $1 \beta$ and IL-18, it is possible to increase our understanding of how different inflammasome inducers affect inflammasome functionality and thus the subsequent immune responses.

\section{Data Availability}

The data used and analyzed in this paper can be obtained from the corresponding authors with reasonable requests.

\section{Conflicts of Interest}

The authors declare that there is no conflict of interest regarding the publication of this paper.

\section{Acknowledgments}

We sincerely thank Kaya Tuerxun for the technical support and Anton Ondracek for his assistance. We also want to thank Dr. Isak Demirel and Dr. Caroline Kardeby for the scientific discussions. This work was supported by the Swedish Knowledge Foundation (Synergi15, ref. no.: 20160044) and Örebro University (strategic grants, ref. nos.: ORU 2.2.14060/2013 and ORU 2018/01219).

\section{Supplementary Materials}

Supplementary Figure 1. Different doses of LPS affects the release of inflammasome cytokines. The concentration of released IL- $1 \beta$ and IL- 18 were detected $24 \mathrm{~h}$ after stimulation of THP-1 cells with ATP, chitosan, and $\mathrm{SiO}_{2}$. Cells were primed with (a) $1 \mu \mathrm{g} / \mathrm{mL}$ or (b) $100 \mathrm{ng} / \mathrm{mL}$ LPS prior to stimulation. The concentration cytokines were normalized against the mean of the control (primed with LPS). Data are shown as mean \pm SD from six individual experiments. Supplementary Figure 2. Dose response of the inflammasome inducers ATP, chitosan, and $\mathrm{SiO}_{2}$. Undifferentiated THP-1 cells were primed with LPS and stimulated with increasing doses of (a) ATP $(\mathrm{mM}),(\mathrm{b})$ chitosan $(\mu \mathrm{g} / \mathrm{mL})$, or (c) $\mathrm{SiO}_{2}(\mu \mathrm{g} / \mathrm{mL})$. Isolated primary monocytes were primed with LPS and stimulated with increasing doses of (d) ATP (M), (e) chitosan $(\mathrm{g} / \mathrm{mL})$, or $(\mathrm{f})$ $\mathrm{SiO}_{2}(\mathrm{~g} / \mathrm{mL})$. The concentration of released IL- $1 \beta$ was measured $24 \mathrm{~h}$ after stimulation. The selected doses are marked with arrows. Data are shown as mean \pm SD from three individual experiments. Supplementary Figure 3. Lack of inflammasome components affect inflammasome-independent cytokines. THP-1 monocytes with functional NLRP3 inflammasome (wt) and inflammasome-deficient THP-1 cells, lacking either functional NLRP3, ASC, or caspase-1 proteins, 
were primed with LPS and stimulated with ATP, chitosan, or $\mathrm{SiO}_{2}$ for $24 \mathrm{~h}$. Controls were treated with LPS alone. The concentration of released (a) IL-6, and (c) TNF were measured. The relative expression of (b) IL-6, and (d) TNF shown is compared to reference genes TBP and HPRT1. Data are shown as mean $\pm \mathrm{SD}$ from six individual experiments. Supplementary Table I. Release of noninflammasome-related cytokine and chemokines. Human inflammatory panel 1 (BioLegend), a multiplex bead-based flow cytometry detection, was used according to the manufacturer's instructions to detect the concentrations of selected cytokines and chemokines from LPS-primed THP-1 cells following stimulation with ATP, chitosan, or $\mathrm{SiO}_{2}$ for $24 \mathrm{~h}$. Some cytokines could not be detected (ND) within the range of the kit. Data are shown as mean from six individual experiments. (Supplementary Materials)

\section{References}

[1] F. Martinon, K. Burns, and J. Tschopp, "The inflammasome: a molecular platform triggering activation of inflammatory caspases and processing of proIL- $\beta$," Molecular Cell, vol. 10, no. 2, pp. 417-426, 2002.

[2] M. S. Dick, L. Sborgi, S. Rühl, S. Hiller, and P. Broz, "ASC filament formation serves as a signal amplification mechanism for inflammasomes," Nature Communications, vol. 7, no. 1, article 11929, 2016.

[3] D. E. Place and T.-D. Kanneganti, "Recent advances in inflammasome biology," Current Opinion in Immunology, vol. 50, pp. 32-38, 2018.

[4] S. Kesavardhana and T.-D. Kanneganti, "Mechanisms governing inflammasome activation, assembly and pyroptosis induction," International Immunology, vol. 29, no. 5, pp. 201-210, 2017.

[5] Y. He, H. Hara, and G. Núñez, "Mechanism and regulation of NLRP3 inflammasome activation," Trends in Biochemical Sciences, vol. 41, no. 12, pp. 1012-1021, 2016.

[6] C. B. A. Grahames, A. D. Michel, I. P. Chessell, and P. P. A. Humphrey, "Pharmacological characterization of ATP- and LPS-induced IL- $1 \beta$ release in human monocytes," British Journal of Pharmacology, vol. 127, no. 8, pp. 1915-1921, 1999.

[7] D. G. Perregaux, P. McNiff, R. Laliberte, M. Conklyn, and C. A. Gabel, "ATP acts as an agonist to promote stimulus-induced secretion of IL-1 beta and IL-18 in human blood," The Journal of Immunology, vol. 165, no. 8, pp. 4615-4623, 2000.

[8] D. Perregaux and C. A. Gabel, "Interleukin-1 beta maturation and release in response to ATP and nigericin. Evidence that potassium depletion mediated by these agents is a necessary and common feature of their activity," The Journal of Biological Chemistry, vol. 269, no. 21, pp. 15195-15203, 1994.

[9] R. Muñoz-Planillo, P. Kuffa, G. Martínez-Colón, B. L. Smith, T. M. Rajendiran, and G. Núñez, " $\mathrm{K}^{+}$efflux is the common trigger of NLRP3 inflammasome activation by bacterial toxins and particulate matter," Immunity, vol. 38, no. 6, pp. 1142-1153, 2013.

[10] D. Fong, P. Grégoire-Gélinas, A. P. Cheng et al., "Lysosomal rupture induced by structurally distinct chitosans either promotes a type 1 IFN response or activates the inflammasome in macrophages," Biomaterials, vol. 129, pp. 127-138, 2017.

[11] C. L. Bueter, C. K. Lee, J. P. Wang, G. R. Ostroff, C. A. Specht, and S. M. Levitz, "Spectrum and mechanisms of inflammasome activation by chitosan," The Journal of Immunology, vol. 192, no. 12, pp. 5943-5951, 2014.
[12] C. L. Bueter, C. K. Lee, V. A. K. Rathinam et al., "Chitosan but not chitin activates the inflammasome by a mechanism dependent upon phagocytosis," The Journal of Biological Chemistry, vol. 286, no. 41, pp. 35447-35455, 2011.

[13] D. M. Gómez, S. Urcuqui-Inchima, and J. C. Hernandez, "Silica nanoparticles induce NLRP3 inflammasome activation in human primary immune cells," Innate Immunity, vol. 23, no. 8, pp. 697-708, 2017.

[14] T. Kusaka, M. Nakayama, K. Nakamura, M. Ishimiya, E. Furusawa, and K. Ogasawara, "Effect of silica particle size on macrophage inflammatory responses," PLoS One, vol. 9, no. 3, article e92634, 2014.

[15] M. Tsugita, N. Morimoto, M. Tashiro, K. Kinoshita, and M. Nakayama, "SR-B1 is a silica receptor that mediates canonical inflammasome activation," Cell Reports, vol. 18, no. 5, pp. 1298-1311, 2017.

[16] C. L. Evavold and J. C. Kagan, "How inflammasomes inform adaptive immunity," Journal of Molecular Biology, vol. 430, no. 2, pp. 217-237, 2018.

[17] C. A. Dinarello, "The biological properties of interleukin-1," European Cytokine Network, vol. 5, no. 6, pp. 517-531, 1994.

[18] C. E. Zielinski, F. Mele, D. Aschenbrenner et al., "Pathogeninduced human TH17 cells produce IFN- $\gamma$ or IL-10 and are regulated by IL-1 $\beta$," Nature, vol. 484, no. 7395, pp. 514-518, 2012.

[19] O. M. Finucane, J. Sugrue, A. Rubio-Araiz, M.-V. Guillot-Sestier, and M. A. Lynch, "The NLRP3 inflammasome modulates glycolysis by increasing PFKFB3 in an IL- $1 \beta$-dependent manner in macrophages," Scientific Reports, vol. 9, no. 1, article 4034, 2019.

[20] K. Deason, T. D. Troutman, A. Jain et al., "BCAP links IL-1R to the PI3K-mTOR pathway and regulates pathogenic Th17 cell differentiation," The Journal of Experimental Medicine, vol. 215, no. 9, pp. 2413-2428, 2018.

[21] K. Nakanishi, T. Yoshimoto, H. Tsutsui, and H. Okamura, "Interleukin-18 is a unique cytokine that stimulates both Th1 and Th2 responses depending on its cytokine milieu," Cytokine \& Growth Factor Reviews, vol. 12, no. 1, pp. 53-72, 2001.

[22] K. Nakanishi, "Unique action of Interleukin-18 on T cells and other immune cells," Frontiers in Immunology, vol. 9, p. 763, 2018.

[23] Y. H. Lee and S.-C. Bae, "Associations between interleukin-1 and IL-1 receptor antagonist polymorphisms and susceptibility to rheumatoid arthritis: a meta-analysis," Cellular and Molecular Biology, vol. 61, no. 8, pp. 105-111, 2015.

[24] S. M. Burm, L. A. N. Peferoen, E. A. Zuiderwijk-Sick et al., "Expression of IL- $1 \beta$ in rhesus EAE and MS lesions is mainly induced in the CNS itself," Journal of Neuroinflammation, vol. 13, no. 1, p. 138, 2016.

[25] Y. Cai, F. Xue, C. Quan et al., "A critical role of the IL- $1 \beta$-IL-1R signaling pathway in skin inflammation and psoriasis pathogenesis," The Journal of Investigative Dermatology, vol. 139, no. 1, pp. 146-156, 2019.

[26] C. A. Dinarello, "Interleukin-1 in the pathogenesis and treatment of inflammatory diseases," Blood, vol. 117, no. 14, pp. 3720-3732, 2011.

[27] S. Dionne, I. D. D’Agata, J. Hiscott, T. Vanounou, and E. G. Seidman, "Colonic explant production of IL-1and its receptor antagonist is imbalanced in inflammatory bowel disease (IBD)," Clinical and Experimental Immunology, vol. 112, no. 3, pp. 435-442, 1998.

[28] Z.-K. Xie, Q.-P. Huang, J. Huang, and Z.-F. Xie, “Association between the IL1B, IL1RN polymorphisms and COPD risk: a 
meta-analysis," Scientific Reports, vol. 4, no. 1, article 6202, 2015.

[29] K. J. Baines, J.-J. Fu, V. M. McDonald, and P. G. Gibson, “Airway gene expression of IL-1 pathway mediators predicts exacerbation risk in obstructive airway disease," International Journal of Chronic Obstructive Pulmonary Disease, vol. 12, pp. 541-550, 2017.

[30] L. Cantarini, G. Lopalco, C. Selmi et al., "Autoimmunity and autoinflammation as the yin and yang of idiopathic recurrent acute pericarditis," Autoimmunity Reviews, vol. 14, no. 2, pp. 90-97, 2015.

[31] C. A. Dinarello, "Overview of the IL-1 family in innate inflammation and acquired immunity," Immunological Reviews, vol. 281, no. 1, pp. 8-27, 2018.

[32] S. Tartey and T.-D. Kanneganti, "Inflammasomes in the pathophysiology of autoinflammatory syndromes," Journal of Leukocyte Biology, vol. 107, no. 3, pp. 379-391, 2020.

[33] T. A. Dowds, J. Masumoto, L. Zhu, N. Inohara, and G. Núñez, "Cryopyrin-induced interleukin lbeta secretion in monocytic cells: enhanced activity of disease-associated mutants and requirement for ASC," The Journal of Biological Chemistry, vol. 279, no. 21, pp. 21924-21928, 2004.

[34] T. T. Pizarro, M. H. Michie, M. Bentz et al., "IL-18, a novel immunoregulatory cytokine, is up-regulated in Crohn's disease: expression and localization in intestinal mucosal cells," Journal of Immunology, vol. 162, no. 11, pp. 6829-6835, 1999.

[35] N. Calvani, H. B. Richards, M. Tucci, G. Pannarale, and F. Silvestris, "Up-regulation of IL-18 and predominance of a Th1 immune response is a hallmark of lupus nephritis," Clinical and Experimental Immunology, vol. 138, no. 1, pp. 171-178, 2004.

[36] H. Konishi, H. Tsutsui, T. Murakami et al., "IL-18 contributes to the spontaneous development of atopic dermatitis-like inflammatory skin lesion independently of IgE/stat 6 under specific pathogen-free conditions," Proceedings of the National Academy of Sciences of the United States of America, vol. 99, no. 17, pp. 11340-11345, 2002.

[37] T. Tanaka, H. Tsutsui, T. Yoshimoto et al., "Interleukin-18 is elevated in the sera from patients with atopic dermatitis and from atopic dermatitis model mice, NC/Nga," International Archives of Allergy and Immunology, vol. 125, no. 3, pp. 236240, 2001.

[38] H. Tanaka, N. Miyazaki, K. Oashi et al., "IL-18 might reflect disease activity in mild and moderate asthma exacerbation," The Journal of Allergy and Clinical Immunology, vol. 107, no. 2, pp. 331-336, 2001.

[39] T. Yoshimoto, H. Tsutsui, K. Tominaga et al., "IL-18, although antiallergic when administered with IL-12, stimulates IL-4 and histamine release by basophils," Proceedings of the National Academy of Sciences of the United States of America, vol. 96, no. 24, pp. 13962-13966, 1999.

[40] Y. Ishikawa, T. Yoshimoto, and K. Nakanishi, "Contribution of IL-18-induced innate T cell activation to airway inflammation with mucus hypersecretion and airway hyperresponsiveness," International Immunology, vol. 18, no. 6, pp. 847-855, 2006.

[41] K. Yasuda, K. Nakanishi, and H. Tsutsui, "Interleukin-18 in health and disease," International Journal of Molecular Sciences, vol. 20, no. 3, p. 649, 2019.

[42] S. D. Brydges, L. Broderick, M. D. McGeough, C. A. Pena, J. L. Mueller, and H. M. Hoffman, "Divergence of IL-1, IL-18, and cell death in NLRP3 inflammasomopathies," The Journal of Clinical Investigation, vol. 123, no. 11, pp. 4695-4705, 2013.
[43] S. Haznedaroglu, M. A. Oztürk, B. Sancak et al., "Serum interleukin 17 and interleukin 18 levels in familial Mediterranean fever," Clinical and Experimental Rheumatology, vol. 23, 4 Suppl 38, pp. S77-S80, 2005.

[44] H. Babaoglu, O. Varan, H. Kucuk et al., "On demand use of anakinra for attacks of familial Mediterranean fever (FMF)," Clinical Rheumatology, vol. 38, no. 2, pp. 577-581, 2019.

[45] G. Cavalli and C. A. Dinarello, "Anakinra therapy for noncancer inflammatory diseases," Frontiers in Pharmacology, vol. 9, article 1157, 2018.

[46] C. A. Dinarello and G. Kaplanski, "Interleukin-18 treatment options for inflammatory diseases," Expert Review of Clinical Immunology, vol. 1, no. 4, 2014.

[47] G. Fenini, E. Contassot, and L. E. French, "Potential of IL-1, IL-18 and Inflammasome inhibition for the treatment of inflammatory skin diseases," Frontiers in Pharmacology, vol. 8, p. 278, 2017.

[48] M. M. Gaidt, T. S. Ebert, D. Chauhan et al., "Human monocytes engage an alternative inflammasome pathway," Immunity, vol. 44, no. 4, pp. 833-846, 2016.

[49] E. Sag, Y. Bilginer, and S. Ozen, “Autoinflammatory diseases with periodic fevers," Current Rheumatology Reports, vol. 19, no. 7, p. 41, 2017.

[50] J. Palomo, D. Dietrich, P. Martin, G. Palmer, and C. Gabay, "The interleukin (IL)-1 cytokine family-balance between agonists and antagonists in inflammatory diseases," Cytokine, vol. 76, no. 1, pp. 25-37, 2015.

[51] F. L. van de Veerdonk and M. G. Netea, "T-cell subsets and antifungal host defenses," Current Fungal Infection Reports, vol. 4, no. 4, pp. 238-243, 2010.

[52] S. Patel and A. Goyal, "Chitin and chitinase: role in pathogenicity, allergenicity and health," International Journal of Biological Macromolecules, vol. 97, pp. 331-338, 2017.

[53] L. Andersson, I. L. Bryngelsson, A. Hedbrant et al., "Respiratory health and inflammatory markers - exposure to respirable dust and quartz and chemical binders in Swedish iron foundries," PLoS One, vol. 14, no. 11, article e0224668, 2019.

[54] H. Westberg, A. Hedbrant, A. Persson et al., "Inflammatory and coagulatory markers and exposure to different size fractions of particle mass, number and surface area air concentrations in Swedish iron foundries, in particular respirable quartz," International Archives of Occupational and Environmental Health, vol. 92, no. 8, pp. 1087-1098, 2019.

[55] C. J. Johnston, K. E. Driscoll, J. N. Finkelstein et al., "Pulmonary chemokine and mutagenic responses in rats after subchronic inhalation of amorphous and crystalline silica," Toxicological Sciences, vol. 56, no. 2, pp. 405-413, 2000.

[56] A. Torres, B. Dalzon, V. Collin-Faure, and T. Rabilloud, "Repeated vs. acute exposure of RAW264.7 mouse macrophages to silica nanoparticles: a bioaccumulation and functional change study," Nanomaterials, vol. 10, no. 2, p. 215, 2020.

[57] G. Cavalli, F. Fallanca, C. A. Dinarello, and L. Dagna, "Treating pulmonary silicosis by blocking interleukin 1," American Journal of Respiratory and Critical Care Medicine, vol. 191, no. 5, pp. 596-598, 2015.

[58] R. L. Schmidt and L. L. Lenz, "Distinct licensing of IL-18 and IL-1 $\beta$ secretion in response to NLRP3 inflammasome activation," PLoS One, vol. 7, no. 9, article e45186, 2012.

[59] J. S. Bezbradica, R. C. Coll, and K. Schroder, "Sterile signals generate weaker and delayed macrophage NLRP3 inflammasome 
responses relative to microbial signals," Cellular \& Molecular Immunology, vol. 14, no. 1, pp. 118-126, 2017.

[60] A. J. Puren, G. Fantuzzi, and C. A. Dinarello, "Gene expression, synthesis, and secretion of interleukin 18 and interleukin 1beta are differentially regulated in human blood mononuclear cells and mouse spleen cells," Proceedings of the National Academy of Sciences of the United States of America, vol. 96, no. 5, pp. 2256-2261, 1999.

[61] Q. Zhu and T.-D. Kanneganti, "Cutting edge: distinct regulatory mechanisms control proinflammatory cytokines IL-18 and IL-1 $\beta$," Journal of Immunology, vol. 198, no. 11, pp. 4210-4215, 2017.

[62] C. M. Cahill and J. T. Rogers, "Interleukin (IL) $1 \beta$ induction of IL-6 is mediated by a novel phosphatidylinositol 3-kinasedependent $\mathrm{AKT} / \mathrm{I} \kappa \mathrm{B}$ kinase $\alpha$ pathway targeting activator protein-1," The Journal of Biological Chemistry, vol. 283, no. 38, pp. 25900-25912, 2008.

[63] L. DiPeso, D. X. Ji, R. E. Vance, and J. V. Price, "Cell death and cell lysis are separable events during pyroptosis," Cell Death Discovery, vol. 3, no. 1, article 17070, 2017. 